\author{
PFC/JA-88-49 \\ X-Ray Imaging Arrays for Impurity Transport \\ Studies on the Texas Experimental Tokamak \\ Kevin W. Wenzel and Richard D. Petrasso
}

September 1989

Accepted for publication in: Revieu of Scientific Instruments

\author{
Plasma Fusion Center \\ Massachusetts Institute of Technology
}

Cambridge, MA 02139 


\title{
X-Ray Imaging Arrays for Impurity Transport Studies on the Texas Experimental Tokamak
}

\author{
Kevin W. Wenzel and Richard D. Petrasso \\ MIT Plasma Fusion Center, Cambridge, MA 02139
}

September 1989

Three multichannel soft $x$-ray imaging arrays are used for impurity transport studies on the Texas Experimental Tokamak. The entire system has a total of 92 broadband $x$-ray detectors that view the plasma at the same toroidal location from three different poloidal angles: Array A is a 40-channel vertically-viewing set of silicon surface barrier diodes (SBDs): Array B is a 40-channel horizontally-viewing set of SBDs; and Array C is a 12-channel set of $p$-intrinsic- $n$ ( $p$ in) detectors viewing the plasma from $45^{\circ}$ above the horizontal plane. Array B is absolutely calibrated, Array A is relatively calibrated in situ, and Array C is cross-calibrated relative to Array B. In this article we describe the primary components of the system: 1) the specific $x$-ray diodes used for the arrays and their spectral sensitivities; 2) the corresponding electronics necessary for data acquisition; and 3) the vacuum system for the arrays. Furthermore we describe a novel krypton edge absorption filter which discriminates between the resonance line radiation of He-like and $\mathrm{H}$-like aluminum injected into TEXT plasmas. 


\section{Introduction}

Soft x-ray emissivity measurements constitute a valuable diagnostic technique for studying impurity transport in laboratory plasmas. For plasmas with electron temperatures exceeding a few hundred electron-volts, $\mathrm{X}$ radiation is produced during electron collisions with ions through the mechanisms of bremsstrahlung, radiative recombination, and line radiation. ${ }^{1-14}$ The intensity of this radiation emitted from a plasma depends on the electron density $\left(n_{e}\right)$, electron temperature $\left(T_{e}\right)$, and the density and ionization states of impurity ions in the plasma. ${ }^{2}$ Measurements of the broadband $x$ emissions can therefore be used to directly determine local plasma parameters.

With the objective of measuring impurity density profiles we can, with independent and simultaneous measurements of $n_{e}(r)$ and $T_{\epsilon}(r)$ as well as some qualitative knowledge of the impurities, begin to infer the level of impurities in the plasma from measurements of broadband $x$-ray brightness profiles. ${ }^{13}$ This method can be refined through the use of multiple arrays of detectors with different absorption filters to yield some spectroscopic resolution, and thus discrimination between different ionization states of a single impurity $y^{9,11}$ or different impurities. ${ }^{10,14}$ In certain circumstances, this information can be used to estimate the average ionic charge $\left(Z_{e f f}\right)$ of the plasma. This is an especially important quantity because it is often directly related to four important properties of the plasma: 1 ) the radiative component of the power balance; 2 ) the current density profile in a tokamak; 3) the extent to which the fuel ions (hydrogen) are diluted; and 4) the efficacy of auxiliary heating methods. In addition to the behavior of impurities intrinsic to the tokamak, we may study general impurity transport by injecting. via laser ablation, ${ }^{15}$ small amounts of known elements into the plasma. ${ }^{16,9,11,17}$ In this sort of experiment confusion over the source of particles is reduced, because the injection is temporally well defined and trace, non gaseous, elements injected by laser ablation are less likely to recycle once they have escaped the bulk plasma. ${ }^{15}$ 
The imaging system described herein was constructed to measure impurity profiles with good spatial and temporal resolution using multiply-filtered arrays on the Texas Experimental Tokamak ${ }^{18}$ (TEXT; minor radius $a=0.26 \mathrm{~m}$, major radius $R_{0}=1.0 \mathrm{~m}$ ). This tokamak is especially well-suited for this type of experiment because of the availability of a large number of independent diagnostics for measuring profiles of many other plasma parameters in addition to the x-ray emission. Of particular importance to impurity transport studies is the availability of time resolved $n_{e}$ and $T_{e}$ profiles for each discharge. The electron density is obtained with a six channel far infrared interferometer, ${ }^{19}$ and the temperature is measured with a ten-channel Electron Cyclotron Emission (ECE) grating polychromator. ${ }^{20}$ The ECE system is calibrated against a single channel ruby laser Thomson scattering diagnostic. ${ }^{21}$ Other important diagnostics include monochromators in the visible through $\mathrm{UV}$ wavelength regions, ${ }^{17}$ an $\mathrm{x}$-ray spectrometer, ${ }^{22}$ and a ten channel bolometer array for measuring time dependent profiles of the total radiated power. ${ }^{23}$

In addition to the application described above, the $\mathrm{x}$-ray imaging arrays on TEXT are useful for studying several other aspects of plasma behavior. For example, the high frequency response ( $\leqslant 100 \mathrm{kHz}$ cutoff) of the system provides an excellent means for examining fast magnetohydrodynamic (MHD) instabilities in the plasma, ${ }^{24,25}$ as well as sawtooth oscillations. ${ }^{8,26}$ These instabilities are clearly manifested through changes in the broadband $\mathrm{x}$-ray signals because of their perturbations on the electron temperature, density, and impurity profiles. A further use of the x-ray imaging system is the tomographic reconstruction of the plasma $x$-ray emissivity from the three poloidal sets of $x$-ray brightness profiles. ${ }^{27-29}$ For this application, the imaging system provides a practical compromise between radial and poloidal resolution; i.e., poloidal modes with mode numbers $m \leq 2$ can be resolved, and the radial resolution is $\delta r \lesssim 2.5 \mathrm{~cm}(\delta r / a \simeq 0.1)$.

This article is organized as follows. Section I describes the major components of the system, including the detectors and their spectral sensitivities, the associated electronics 
for signal processing and data acquisition, and the vacuum system. A description of a novel krypton gas edge absorption filter is given in Section II along with some preliminary data from aluminum injection experiments.

\section{X-Ray Imaging System Components}

Figure 1 shows a schematic of the complete $\mathrm{x}$-ray imaging system as configured on TEXT. The entire system consists of a total of $92 \mathrm{x}$-ray detectors, distributed among two multichannel arrays of silicon surface barrier (SBD) x-ray detectors and one array of $p$ intrinsic-n (pin) diodes. The two SBD arrays are Array A (Fig. 2a), a vertically-viewing 40-channel array, and Array B (Fig. 2b), a horizontally-viewing 40-channel array. Array B, is arranged in two columns of 20 detectors each. These two columns form separate arrays viewing the same plasma and may be filtered differently. Array C (Fig. 2c) is a 12-channel pin array located at an angle of $45^{\circ}$ between the vertical and the horizontal midplane. Array A views chords across the entire plasma cross section from $-26 \mathrm{~cm}$ to $+26 \mathrm{~cm}$ (inside to outside) with a spatial resolution of $\simeq 1.3 \mathrm{~cm}$. Array B views chords from -23 $\mathrm{cm}$ to $-25 \mathrm{~cm}$ (bottom to top) with a spatial resolution of $\simeq 2.4 \mathrm{~cm} .{ }^{30}$ Array $\mathrm{C}$ views only the top one-quarter of the plasma from $0 \mathrm{~cm}$ to $+15 \mathrm{~cm}$ with a spatial resolution of $\simeq 1.3$ $\mathrm{cm}$. All three arrays view the plasma from the same toroidal location, and each is housed in a stainless steel vacuum enclosure that is separated from the TEXT vacuum system by a beryllium window $25 \mu \mathrm{m}(1 \mathrm{mil})$ thick. This window also completely attenuates visible plasma light to which the detectors are also sensitive.

An original version of Array A was installed on TEXT several years ago, and is described in detail elsewhere. ${ }^{31}$ In the context of the work presented here, three major improvements have been made to this array: ${ }^{32}$ 1) an in situ relative calibration of the rectangular diodes against absolutely calibrated $\operatorname{diodes}^{1}$ (the response of the Array A diodes was measured relative to the response of round diodes that could be positioned adjacent to any Array A diode, as shown in Fig. 2a); 2) the modification of the vacuum 
box to allow the entire plasma cross section to be viewed $( \pm 26 \mathrm{~cm}$ instead of $\pm 21.5 \mathrm{~cm}$ previously); and 3) the addition of the beryllium window to physically divide the array vacuum system from that of TEXT.

\section{A. X-Ray Detectors and Spectral Response}

The detectors in Array A are p-type SBDs, those in Array B are n-type, and the detectors used in Array $\mathrm{C}$ are $p$-intrinsic- $n$ diodes. All three types of detectors are widely used for the detection of x-rays from plasma confinement experiments (see especially Table I of Ref. 1). The smaller pin diodes were chosen for Array $\mathrm{C}$ because of size constraints imposed by the TEXT torque frame. Small diodes from United Detector Technology (model A4V-12PL) ${ }^{33}$ are sufficiently compact for use in this experiment, and are similar to those diodes used for $\mathrm{x}$-ray tomography experiments on Alcator- $\mathrm{C}^{27}$ and the Missouri Mirror Experiment. ${ }^{34}$ The total size of a set of 12 pin detectors is approximately one inch long by one-quarter inch wide, as shown in Fig. 2c. This size makes these detectors ideal for use in a small vacuum box. The advantages of small size and low cost of the pin diodes would not necessarily justify their use for all three arrays on TEXT; the geometry of Array $\mathrm{C}$ results in a small solid viewing angle. (The detector active area $A_{d}=0.152 \mathrm{~cm} \times 0.279 \mathrm{~cm}$ $=0.042 \mathrm{~cm}^{2}$, the detector-to-slit distance $L_{s}=8.6 \mathrm{~cm}$, and the detector-to-plasma distance $L_{p}=69.6 \mathrm{~cm}$.) This has the consequence of a small response signal, and the signal-to-noise ratio is smaller for Array C than it is for Array A $\left(A_{d}=0.75 \mathrm{~cm}^{2}, L_{s}=44.0 \mathrm{~cm}\right.$, and $L_{p}=$ $122.6 \mathrm{~cm})$ or Array B $\left(A_{d}=0.5 \mathrm{~cm}^{2}, L_{s}=17.0 \mathrm{~cm}\right.$, and $\left.L_{p}=70.5 \mathrm{~cm}\right)$. The noise from all three arrays is dominated by the detector/preamplifier combination, rather than detector response to extraneous radiation. With $1 \mathrm{M} \Omega$ feedback resistors typical signal/noise levels from the preamplifiers are, for Array $\mathrm{A}, 0.1 \mu \mathrm{A} / 0.003 \mu \mathrm{A}$; from Array $\mathrm{B}, 0.65 \mu A / 0.02 \mu A$; and from Array C, $0.08 \mu . A / 0.02 \mu A$

Severe size restrictions, as mandated for Array C, were not imposed on the vacuum boxes for Arrays $\mathrm{A}$ and B. For these arrays larger SBD detectors were used. The detec- 
tors in Array A were Ortec model ARXX-3X25-100S partially-depleted SBDs, ${ }^{31,35}$ those in Array B were Tennelec model PD-50-100-17 partially depleted SBDs. ${ }^{36}$ The Tennelec diodes have been absolutely calibrated as described in Ref. 1.

Despite physical differences in their front surfaces, the diodes have similar low energy spectral responses in the $x$-ray wavelength range when they view the plasma through the $25 \mu \mathrm{m}$ beryllium window. This is because the low-energy response is dominated by the beryllium absorption, not attentuation by the detector surface material. The front of the Ortec diodes (Array A) consists of $\sim 2500 \AA$ aluminum, that of the Tennelec diodes (Array B) is $\sim 250 \AA$ of gold, and that of the UDT diodes (Array C) is $\sim 700 \AA$ of $\mathrm{SiO}_{2}$. On the high energy side of the spectrum, the response of all the diodes is nearly the same because it is determined predominantly by the total thickness of the silicon used for the detectors, not the depletion depth. ${ }^{1}$ The detectors in Arrays A, B and C have physical thicknesses $\sim 300 \mu \mathrm{m}, \sim 400 \mu \mathrm{m}$, and $\sim 250 \mu \mathrm{m}$ respectively. The total response for all three arrays when they view the plasma through the beryllium window is shown in Fig. 3. From this figure, it is seen that they all have nearly identical responses over the energy. range of interest (i.є., $\sim 1 \mathrm{keV}$ to $20 \mathrm{keV}$ ). Indeed the brightness profiles measured by the three arrays agree to within $\lesssim 10 \%$ when they are filtered with only their permanent front surfaces and the intervening $25 \mu \mathrm{m}$ Be. Similarity in the detector responses is particularly important for the application of x-ray tomography.

\section{B. Signal Processing and Data Acquisition System}

The signal current from the detectors (proportional to the total incident $\mathrm{x}$-ray power) is converted to a volt age signal in preamplifier boards directly adjacent to the array vacuum boxes. The signal is then amplified and analog filtered in the tokamak enclosure before being sent to the TEXT data room for digitization. This overall signal processing scheme is shown schematically in Fig 4a. Array A has slightly different electronics ${ }^{31}$ than Arrays B and $\mathrm{C}$; the discussion below is limited to the electronics of Arrays B and C. The general 
characteristics of all three arrays are, however, quite similar.

The detector signal currents, typically on the order of a microamp for the Array B central channel and decreasing rapidly for channels viewing chords farther from the plasma center, are transmitted via Physicon vacuum feedthroughs to the AC-coupled preamplifier stage illustrated in Fig. 4b. An AC-coupled preamp design was used in order to allow the bias voltage for the Array B diodes to be easily varied over a wide range, up to the maximum suggested by the diode manufacturer $(\sim+70 \mathrm{~V})$. The diodes of Array $\mathrm{C}$ are simply biased at a constant $-15 \mathrm{~V}$ taken from the power supply leg of the operational amplifier; this avoids the need for a separate power and bias voltage supply for this array. (The Array B diodes [n-type silicon] require a positive bias voltage, but the Array C diodes [p-type silicon] require a negative bias voltage.) In the preamps for all the arrays, the signal current is converted to a voltage by the $1 \mathrm{M} \Omega$ feedback resistor across an Analog Devices AD-544 operational amplifier. This provides a gain of $1 \mathrm{~V} / \mu \mathrm{A}$, so signal levels at the output of the preamplifier are typically a few volts for the central channels. (The profiles of chordintegrated signals are approximately Gaussian, similar to the temperature profile, with an $\mathrm{e}^{-1}$ width varying from $3 \mathrm{~cm}$ to $10 \mathrm{~cm}$. This width depends strongly on the combination of plasma current and toroidal magnetic field confining the plasma. ${ }^{39}$ )

After the preamplifier stage, the signals are passed through twinax cables to amplifier modules with variable gains ranging from 1 to 400 . By changing the amplifier gain the user can easily compensate for the different signal levels between array channels and provide adequate signal levels for digitization. (The digitizer input voltage limits are $\pm 5 \mathrm{~V}$.) A schematic of the two-stage amplifier circuit is shown in Fig. 4c. The frequency responses of the preamplifier and amplifier, determined using a sine wave input from a signal generator, are shown in Figs. 5a and $5 \mathrm{~b}$ respectively. This figure shows that the overall response of the preamplifier/amplifier combination is constant up to about $20 \mathrm{kHz}$, depending on the amplifier gain. (In practice, we rarely run with any gains exceeding 25). 
After amplification the signals are filtered by variable-frequency/variable-gain filters. These modules have high frequency ( $3 \mathrm{db}$ ) cutoffs of $2,4,8,20$, and $40 \mathrm{kHz}$, and a variable gain of either \pm 1 or $\pm \frac{1}{2}$. The filter frequency is normally chosen to coincide with half the digitization frequency in order to satisfy the Nyquist sampling theorem and thereby reduce the chance of frequency aliasing.

The signal is converted from the analog output of the filter module to digital data by a DSP Technology Transiac Traq I system. ${ }^{40}$ This system is capable of digitizing at a rate up to $100 \mathrm{kHz}$ with 12 -bit resolution. For normal TEXT runs, the signal is digitized at $20 \mathrm{kHz}$. This frequency is sufficient to resolve sawtooth and MHD activity because the sawtooth frequency ranges between $150 \mathrm{~Hz}$ and $1 \mathrm{kHz}$ and the characteristic MHD frequency is $\sim 5$ $\mathrm{kHz}$. For experiments designed specifically to study fast MHD oscillations, the data are digitized at $50 \mathrm{kHz}$.

\section{Vacuum Systems}

As previously mentioned, each array is housed in a stainless steel box separated by a $25 \mu \mathrm{m}$ thick beryllium window from the main vacuum of TEXT (nominally $\sim 10^{-8}$ torr after discharge cleaning ${ }^{18}$ ). This window is simply glued with Torr seal epoxy ${ }^{41}$ over a narrow slit cut into each array's mounting flange. An advantage of separating the tokamak and array vacuums is that a dedicated pump is then unnecessary to maintain high vacuum in the array boxes. An additional advantage is that the boxes may be easily backfilled with various gases for use as $\mathrm{x}$-ray filters. A specific example of this technique, using krypton as an $\mathrm{x}$-ray filter, is described in the following section.

\section{Krypton Filtering and Preliminary Results}

In order to measure the charge state distribution and transport properties of aluminum injected into TEXT we have developed a set of filters for discrimination between $\mathrm{x}$ radiation from the three highest charge states of aluminum. (He-like $\mathrm{Al}^{+11}, \mathrm{H}-$ like $\mathrm{Al}^{+12}$ and fully 
stripped $\mathrm{Al}^{+13}$ are the predominant three charge states inside the edge plasma.) The three filters used for this purpose are Filter A, consisting of $4.6 \mathrm{mg} / \mathrm{cm}^{2}(25 \mu \mathrm{m})$ Be alone; Filter G, including $4.6 \mathrm{mg} / \mathrm{cm}^{2}$ Be plus $1.52 \mathrm{mg} / \mathrm{cm}^{2}$ krypton; and Filter D, composed of 4.6 $\mathrm{mg} / \mathrm{cm}^{2}$ Be plus $30.5 \mathrm{mg} / \mathrm{cm}^{2}$ carbon. The $\mathrm{x}$-ray spectral response for all three of these filters is shown in Fig. 6. For typical TEXT temperatures Filter A responds primarily to the strong $2 \mathrm{p}$-ls resonance transition lines from both He-like and H-like aluminum at 1.61 $\mathrm{keV}$ and $1.73 \mathrm{keV}$ respectively. However Filter G responds mostly to the He-like line at 1.61 $\mathrm{keV}$ because of the strong L-shell absorption edge in krypton that begins at $1.675 \mathrm{keV}^{38}$ Thus this filter performs the quantitative discrimination between the He-like and H-like charge state densities. Filter D cuts out all the lines of both the He-like (binding energy $\chi=2.09 \mathrm{keV})$ and $\mathrm{H}$-like $(\chi=2.30 \mathrm{keV})$ states due to its low energy cutoff at about $3 \mathrm{keV}$. This filter is therefore responsive mainly to the continuum from radiative recombination into the ground state of the fully stripped aluminum for the plasma conditions in TEXT. ${ }^{9}$ An important feature of this filter combination is that the difference between the Filter G and Filter D responses is due only to the small window around $1.5 \mathrm{keV}$. Thus this difference signal is strongly dominated by the He-like aluminum contribution.

Typical time histories of central chord-integrated x-ray signals are shown in Fig. 7 for an ohmically heated hydrogen discharge with a toroidal field $B_{T}=2.8 \mathrm{~T}$, plasma current $I_{p}=320 \mathrm{kA}$, and a central line-averaged electron density $\bar{n}_{e}=6.3 \times 10^{13} \mathrm{~cm}^{-3}$. In this particular shot aluminum was injected, using the TEXT laser ablation system, ${ }^{42}$ at 325 $\mathrm{ms}$ in order to study its charge state distribution and transport using the three differently filtered arrays described above: one horizontal channel with only the beryllium window (Array B, Filter A), another horizontal channel with an additional $400 \mu \mathrm{m}$ carbon (Array B, Filter D), and a vertical channel with the $25 \mu \mathrm{m}$ beryllium and $44.0 \mathrm{~cm}$ of krypton at a pressure of 7 torr (Array A, Filter G). Note that the response of Filter A to the aluminum is about 7 times that of Filter $\mathrm{G}$ and 100 times that of Filter D. (Again, when all three 
arrays are filtered the same, their responses are identical within $\sim 10 \%$.) The difference between the Filter $G$ response and the Filter D response to the aluminum injection is largely due to the contribution of the He-like charge state.

Radial brightness profiles taken at $372 \mathrm{~ms}$ during the same discharge, after subtracting the pre-injection signal levels, are shown in Fig. 8. The data from Array A (squares) is shifted outward with respect to the Array B data because of the normal horizontal shift of the plasma column (the Shafronov shift) ${ }^{43}$ due to the toroidal plasma equilibrium. The imaging system thus gives a direct measurement of the position of the plasma center which has been used to correct magnetic measurements of the plasma position. There is no, apparent vertical shift of the plasma as reflected by the profiles from Array B, and all the profiles from Arrays A and B are approximately symmetric about their respective centers. An important conclusion regarding the degree of aluminum ionization that can be drawn from comparison of the shapes of the profiles taken with Filters A and $G$ is that He-like aluminum is the dominant charge state in this discharge, because both filters give profiles of nearly identical shape. If, on the other hand, the $\mathrm{H}$-like state were dominant in the central hot region of the plasma the Filter $G$ profile would be significantly broader than that from Filter A. This is because the He-like ions (again, the dominant contributor to the signal of Filter $G$ ) would be depleted in the central region. More quantitative analysis is underway to directly determine the absolute density profiles of all three aluminum charge states.

In summary, we have described three arrays of broadband, soft x-ray detectors used on TEXT for examining impurity transport. Array A is a 40-channel, vertically-viewing SBD system. Array B is a 40-channel, horizontally-viewing SBD system separated into two columns, each of which may be independently filtered. Array C is a 12-channel pin system viewing the upper quarter of the plasma from $45^{\circ}$ between the horizontal and vertical planes. All three arrays view a cross section of the plasma at the same toroidal location. 
These arrays are separated from the main TEXT vacuum by $25 \mu \mathrm{m}$ beryllium windows, alleviating the need for a dedicated pump for each array and allowing the array vacuum boxes to be backfilled with different gases for x-ray filtering. The Be window also serves to filter unwanted visible, ultraviolet and vacuum ultraviolet radiation from the plasma. In the context of impurity profile measurements, it is important to avoid this radiation because the abundance of lines in the ultraviolet spectrum, to which the detectors are sensitive, makes quantitative interpretation difficult.

The complete set of three arrays is unique in part because the Array B diodes have been absolutely calibrated. The Array A diodes have been relatively calibrated in situ against scanning, absolutely calibrated diodes (see Fig. 2a) and also against Array B. The complete system of 92 detectors in three arrays provides a significant improvement over the original TEXT x-ray imaging system ${ }^{31}$ in spatial and spectral resolution. Such modifications make possible improved measurements of impurity transport in TEXT. In the context of these studies we have developed a set of three filters to simultaneously measure the absolute density profiles of the three highest charge states of aluminum injected into the tokamak. The krypton edge-absorption filter has so far given qualitative discrimination between the He-like and H-like charge states. In addition to the impurity transport work, fast MHD oscillations can be studied with the system, ${ }^{24,25}$ the data from which can be tomographically inverted to give images of the local x-ray emissivity over the poloidal cross section. $^{28}$

\section{Acknowledgments}

We gratefully acknowledge the invaluable assistance of both the TEXT and Alcator technical staffs. In particular, Tom Herman of TEXT was instrumental in the design and construction of the vacuum systems. We thank Joe Bryan, Steve Hilsberg and Paul Landers of TEXT for their helpful suggestions regarding the design of the electronics. Joe Bosco and Bill Parkin of Alcator kindly provided filter modules modified to allow higher 
cutoff frequencies than previously available. Dr. Robert Granetz generously loaned us the Traq I digitizer system, previously used on Alcator-C. Martha Baker and Dr. Ken Gentle assisted in the design of the vertical array box. We also appreciate the support of Dr. Ron Bravenec, Dr. Steve McCool, Dr. William Rowan, Dr. Alan Wootton, Mark Foster and Brackin Smith at TEXT. This work was supported in part by DOE under Contract Nos. DE-AC02-78ET-51013 and DE-FG05-88ER-53267. 


\section{References}

1. K. W. Wenzel and R. D. Petrasso, Rev. Sci. Instrum. 591380 (1988); and references cited therein.

2. T. F. Stratton, in Plasma Diagnostic Techniques, eds. R. H. Huddlestone and S. L. Leonard, Academic Press, 1965.

3. E. D. Fredrickson, K. McGuire, B. Grek, K.-I. Hattori, D. Johnson, and A. W. Morris, Rev. Sci. Instrum. 591797 (1988).

4. R. B. Spielman, W. W. Hsing, and D. L. Hanson, Rev. Sci. Instrum. 591804 (1988).

5. R. T. Snider, R. Evanko, and J. Haskovev, Rev. Sci. Instrum. 591807 (1988).

6. A. Holland, R. J. Fonck, E. T. Powell, and S. Sesnic, Rev. Sci. Instrum. 591819 (1988).

7. J. P. Anthes, Rev. Sci. Instrum. 591846 (1988).

8. S. von Goeler, W. Stodiek, and N. Sauthoff, Phys. Rev. Lett. 331201 (1974).

9. R. D. Petrasso, F. H. Seguin, N. G. Loter, E. Marmar, and J. Rice, Phys. Rev. Lett. 491826 (1982).

10. R. D. Petrasso, D. J. Sigmar, K. W. Wenzel, J. E. Hopf, M. Greenwald, J. L. Terry, and J. Parker, Phys. Rev. Lett. 57707 (1986).

11. K. W. Wenzel, M. E. Austin, R. D. Petrasso, B. Richards, and W. L. Rowan, Bull. Am. Phys. Soc. 332026 (1988).

12. S. von Goeler, W. Stodiek, H. Eubank, H. Fishman, S. Grebenschikov, and E. Hinnov, Nucl. Fus. 15301 (1975). 
13. R. D. Petrasso, M. Gerassimenko, F. H. Seguin, R. Krogstad, and E. S. Marmar, Nucl. Fus. 21881 (1981).

14. A. Weller, D. Pasini, A. W. Edwards, R. D. Gill, and R. S. Granetz, "Modelling of Soft X-Ray Emission From JET Plasmas," in Conference on Atomic Collisions in Fusion, unpublished, Oxford (1987).

15. E. S. Marmar, J. L. Cecchi, and S. A. Cohen, Rev. Sci. Instrum. 461149 (1975).

16. S. A. Cohen, J. L. Cecchi, and E. S. Marmar, Phys. Rev. Lett. 351507 (1975).

17. W. K. Leung, W. L. Rowan, J. C. Wiley, R. V. Bravenec, K. W. Gentle, W. L. Hodge, D. M. Patterson, P. E. Phillips, T. R. Price, and B. Richards, Plasma Phys. and Cont. Fusion 281753 (1986).

18. K. W. Gentle, Nucl. Technology/Fusion 1479 (1981).

19. TEXT User Handbook, 7th ed., University of Texas Fusion Research Center, unpublished (1985).

20. M. E. Austin, R. Gandy, and K. W. Gentle, Bull. Am. Phys. Soc. 311574 (1986).

21. J. L. Porter, P. E. Phillips, S. C. McCool, S. B. Kim, D. W. Ross, W. H. Miner, J. C. Wiley, Nucl. Fus. 27205 (1987).

22. M. R. Bizak, B. Richards, W. L. Rowan, J. C. Wiley, and H. S. Seung, Bull. Am. Phys. Soc. 281034 (1983).

23. Y. He and R. V. Bravenec, University of Texas FRC Report \# 305 (1988).

24. J. Chen, M. S. Foster, S. C. McCool, A. J. Wootton, and X. Y. Yu, Bull. Am. Phys. Soc. 332023 (1988). 
25. P. M. Schoch, K. A. Connor, T. P. Crowley, J. W. Heard, R. L. Hickock, S. C. McCool, V. Simcic, B. A. Smith, A. J. Wootton, and Y. Z. Yang, 16th European Conference on Controlled Fusion and Plasma Physics, Venice, 1989, to be published.

26. B. B. Kadomtsev, Sov. J. Plasma Phys. 1389 (1975).

27. J. F. Camacho and R. S. Granetz, Rev. Sci. Instrum. 57417 (1986).

28. B. A. Smith, R. S. Granetz, K. W. Wenzel, S. C. McCool, and M. S. Foster, Bull. Am. Phys. Soc. 332025 (1988).

29. R. S. Granetz and P. Smeulders, Nucl. Fus. 28457 (1988).

30. Although the total number of detectors in Arrays A and B is the same, they have different spatial resolution because Array B has two independent 20-channel arrays that view the same poloidal angles. Thus the distance between adjacent chords for Array B is about twice that of Array A. See Fig. 2 for the major differences.

31. J. L. Snipes, Ph.D. thesis, University of Texas at Austin, Physics Dept., FRC Report \# 275. (August 1985).

32. K. W. Wenzel, R. D. Petrasso, J. E. Hopf, S. C. McCool, and M. S. Foster, Bull. Am. Phys. Soc. 311574 (1986).

33. United Detector Technology; Hawthorne California.

34. H. K. Tseng, C. D. Eshelman, T. J. Dolan, E. Leal-Quiros, R. Hane, J. Javedani, and H. Prelas, Bull. Am. Phys. Soc. 332054 (1988).

35. EG\&G Ortec Nuclear Instruments and Systems catalog, pp 86-87, Oak Ridge; Tennessee. 
36. Tennelec Silicon Charged Particle Detector catalogue No. DO-986-3.0k, Oak Ridge, Tennessee.

37. B. L. Henke, P. Lee, T. J. Tanaka, R. L. Shimabukuro, and B. J. Fujikawa, Atomic Data and Nuclear Data Tables 271 (1982); these cross sections were applied for the energy range $30 \mathrm{eV}$ to $1 \mathrm{keV}$.

38. W. H. McMaster, N. Kerr Del Grande J. H. Mallet, and J. H. Hubbel, UCRL-50174SEC2-R1 (1969); these cross sections were applied for energies above $1 \mathrm{keV}$.

39. S. C. McCool, R. V. Bravenec, D. L. Brower, M. S. Foster, K. W. Gentle, S. B. Kim, S. K. Kim, N. C. Luhmann, Jr., D. M. Patterson, W. A. Peebles, P. E. Phillips, B. Richards, W. L. Rowan, B. A. Smith, K. W. Wenzel, and A. J. Wootton, Bull. Am. Phys. Soc. 321842 (1987).

40. DSP Technology Inc., Freemont California (1987).

41. Varian Associates Inc., Vacuum Products Div., Lexington MA.

42. D. R. Terry, W. L. Rowan, W. J. Connally, and W. K. Leung, Symp. Fusion Eng. 2 $1959(1983)$.

43. V. S. Mukhovatov and V. D. Shafronov, Nucl. Fus. 11605 (1971). 


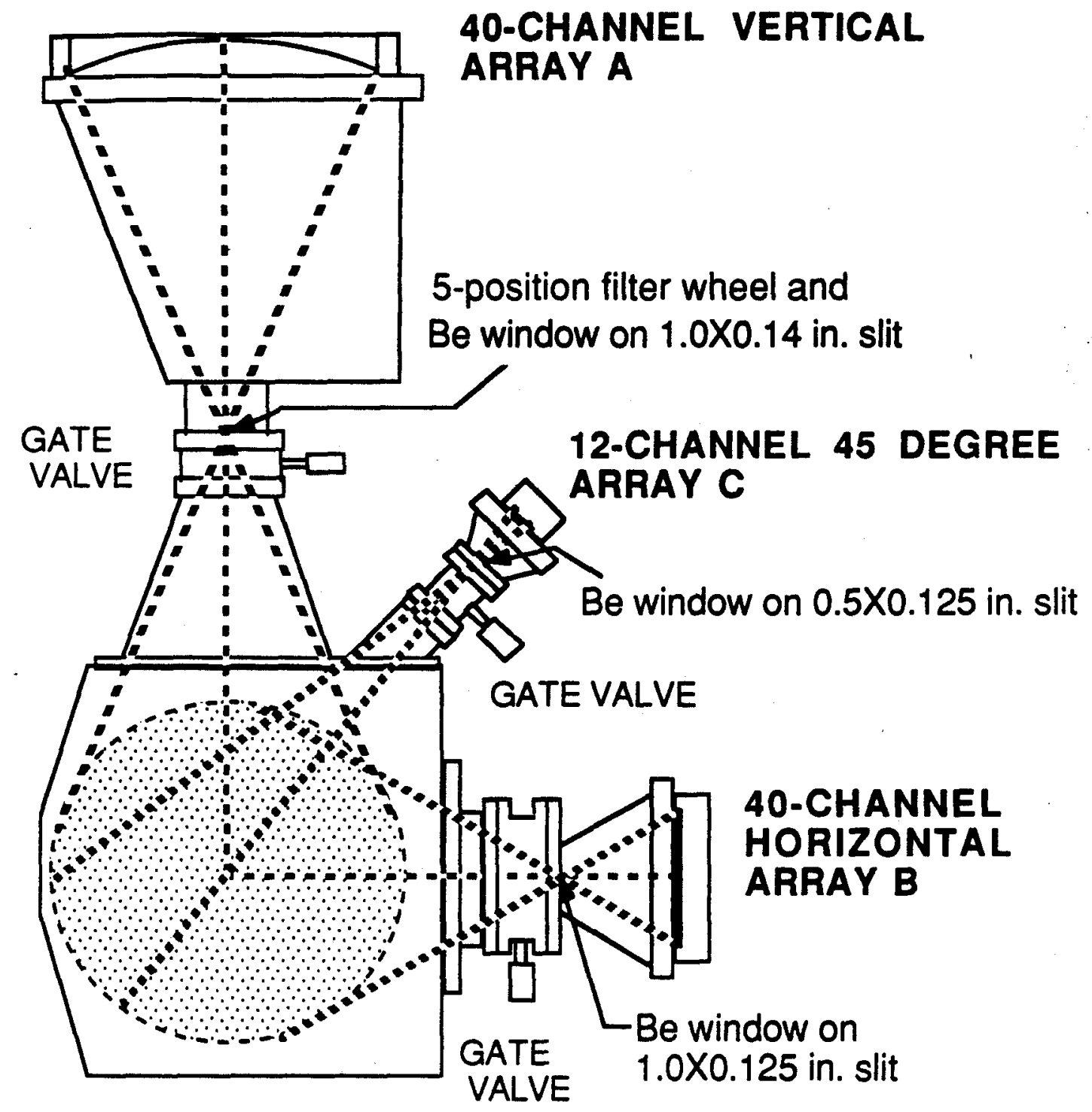

Fig. 1. The TEXT soft $\mathrm{x}$-ray imaging system has a total of 92 detectors in three separate arrays viewing poloidal cross sections of the plasma at the same toroidal location. Array A is a rebuilt version of the 40 -channel SBD array that was originally installed. ${ }^{31}$ Array $B$ is a 40-channel SBD array divided into two 20-channel columns that may be independently filtered. Array C, a 12-channel pin diode array, views only the top quarter of the plasma from a $45^{\circ}$ angle. All three arrays are separated from the main TEXT vacuum by $25 \mu \mathrm{m}$ ( 1 mil) thick beryllium windows. See Fig. 2 for a detailed picture of the individual arrays. (The long dimension of each slit is in the toroidal direction, and the plasma minor radius is $26 \mathrm{~cm}$.) 
Fig. 2. a) Array A, the 40-channel, vertically viewing set of rectangular SBDs. Note the two round SBDs in square white polyethylene holders that can be positioned adjacent to any rectangular main array diode for relative calibration during a TEXT discharge. (The scanning diodes are stationary during a given discharge.) b) Array B, the 40-channel, horizontally viewing set of SBDs. The two columns of 20 detectors each may be independently filtered. (The four holes on each side of the column of detectors are to allow vacuum pumping in the section of the box behind the solid polyethylene detector support structure.) c) Array (C, the 12-channel set of pin detectors that views the plasma from a $45^{\circ}$ angle. (Again the large holes are to allow both sections of the box to be pumped out.) Note the different scales on the three figures; the ruler in all three photographs is six inches long. 


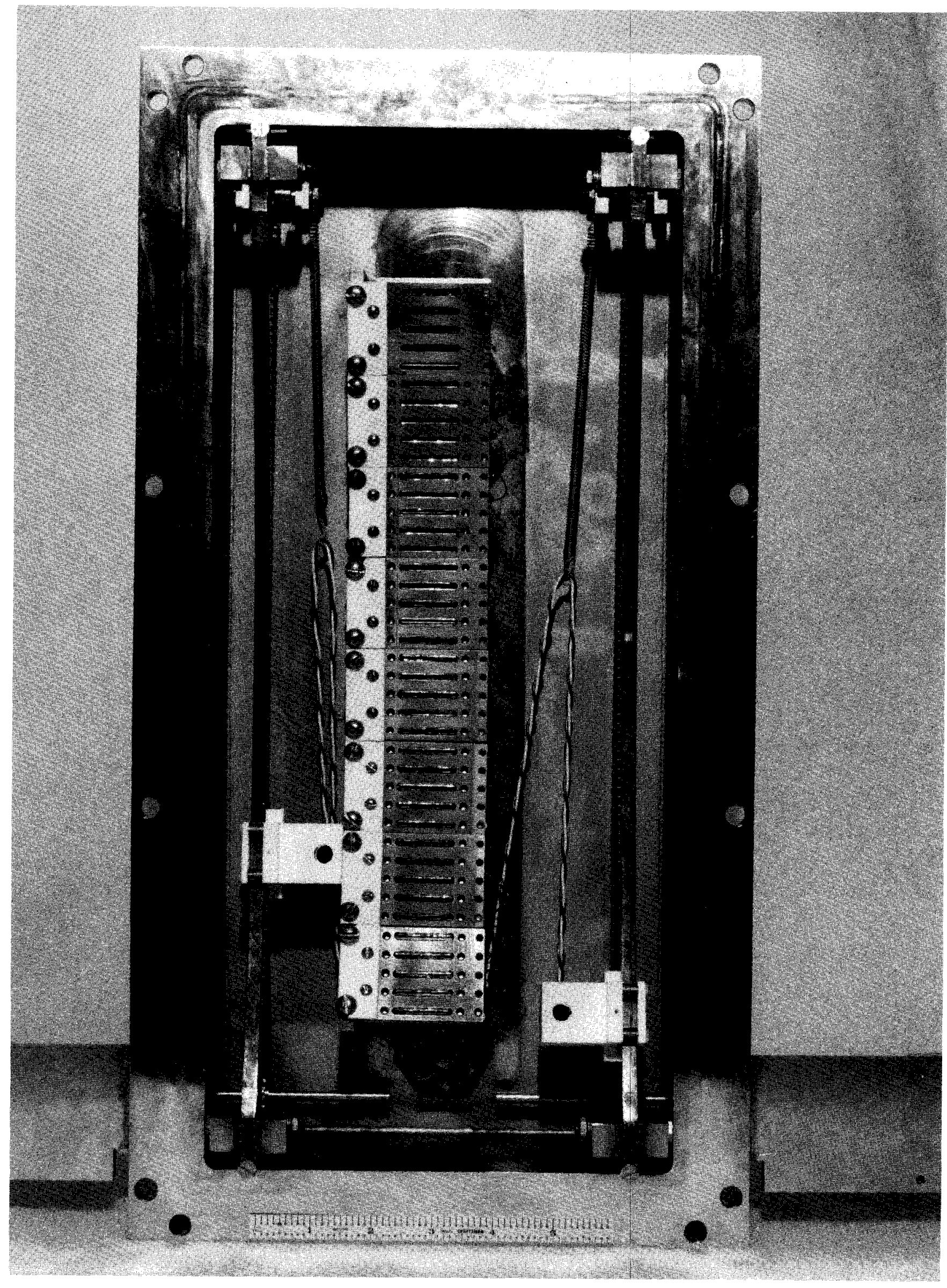




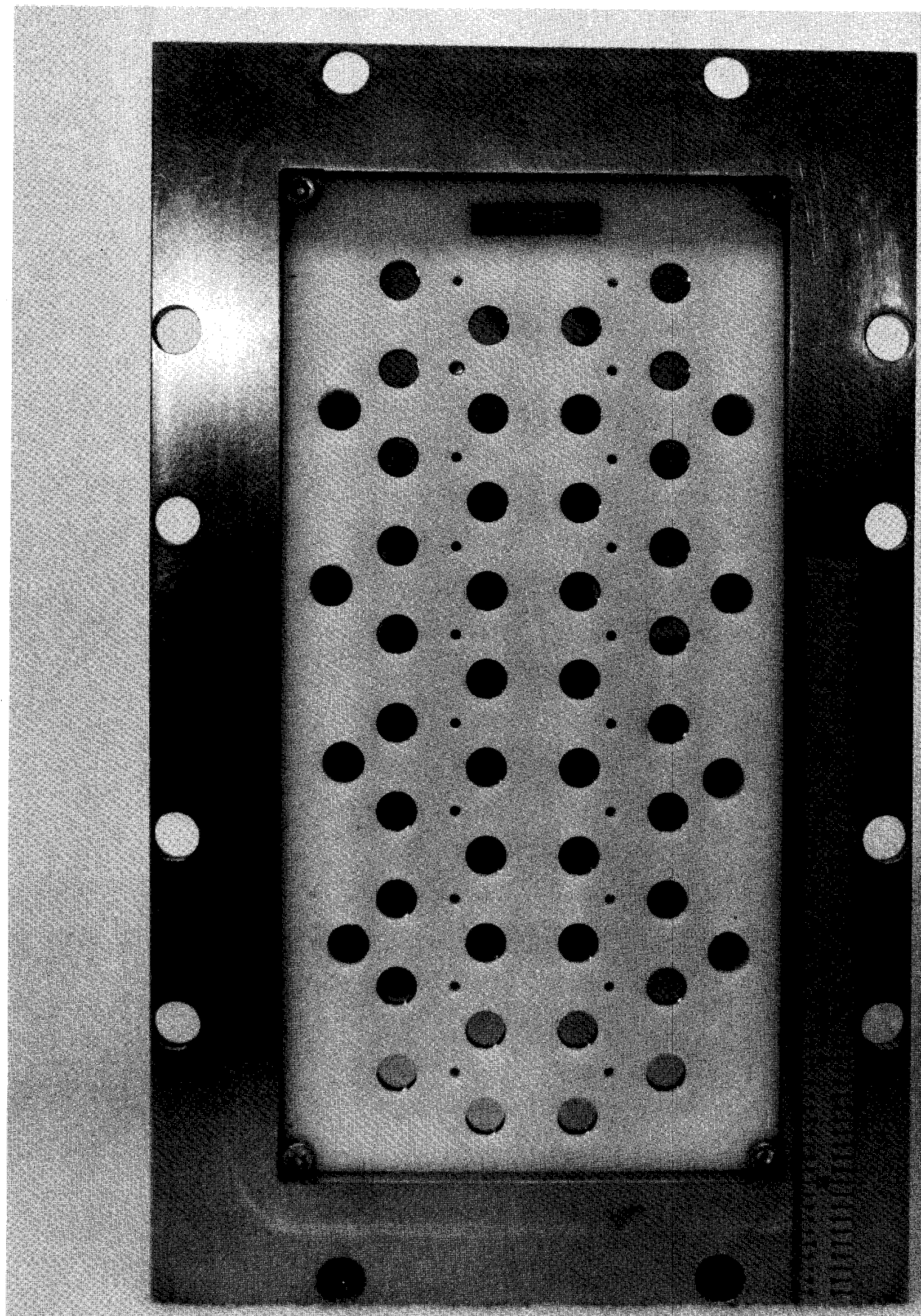




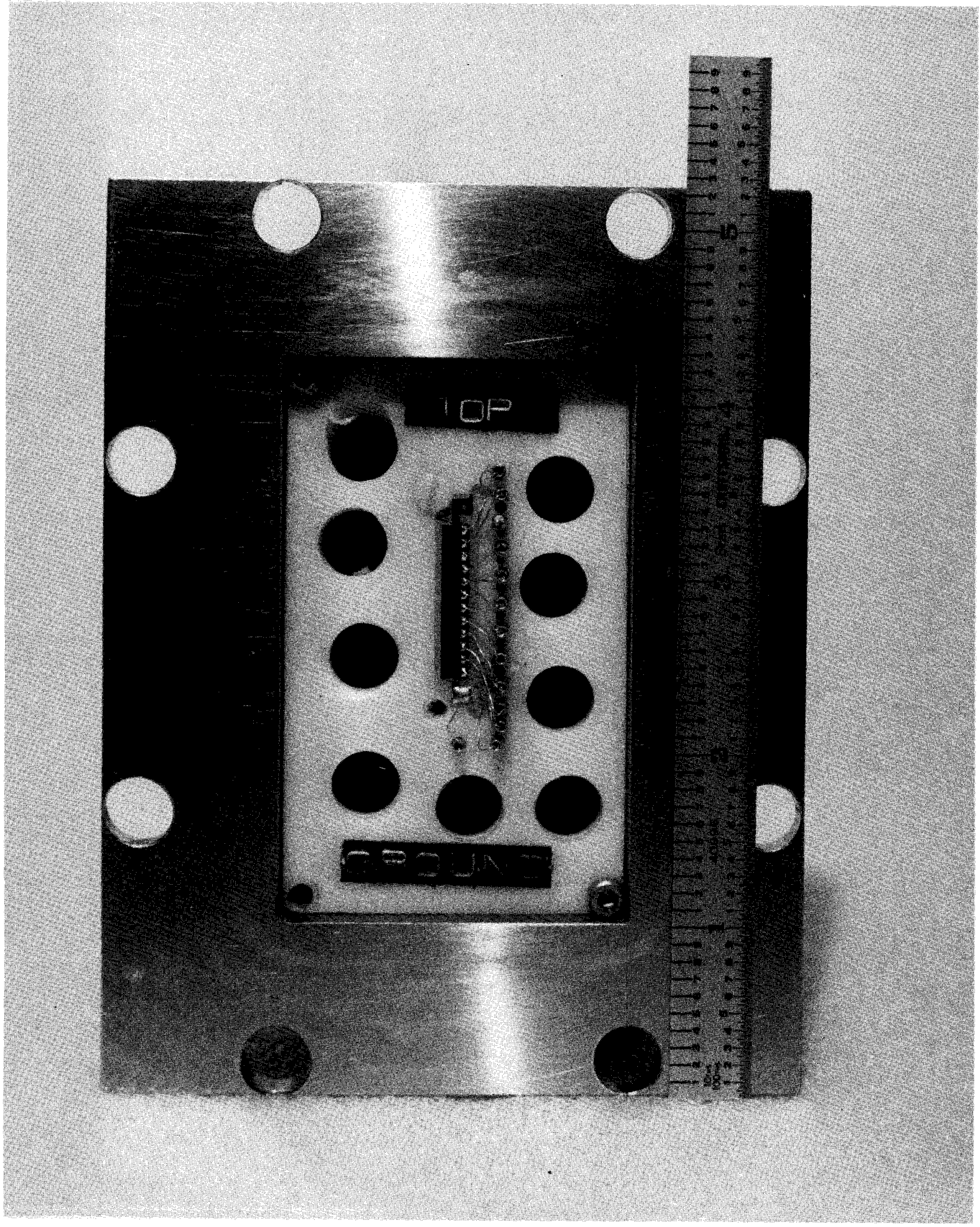




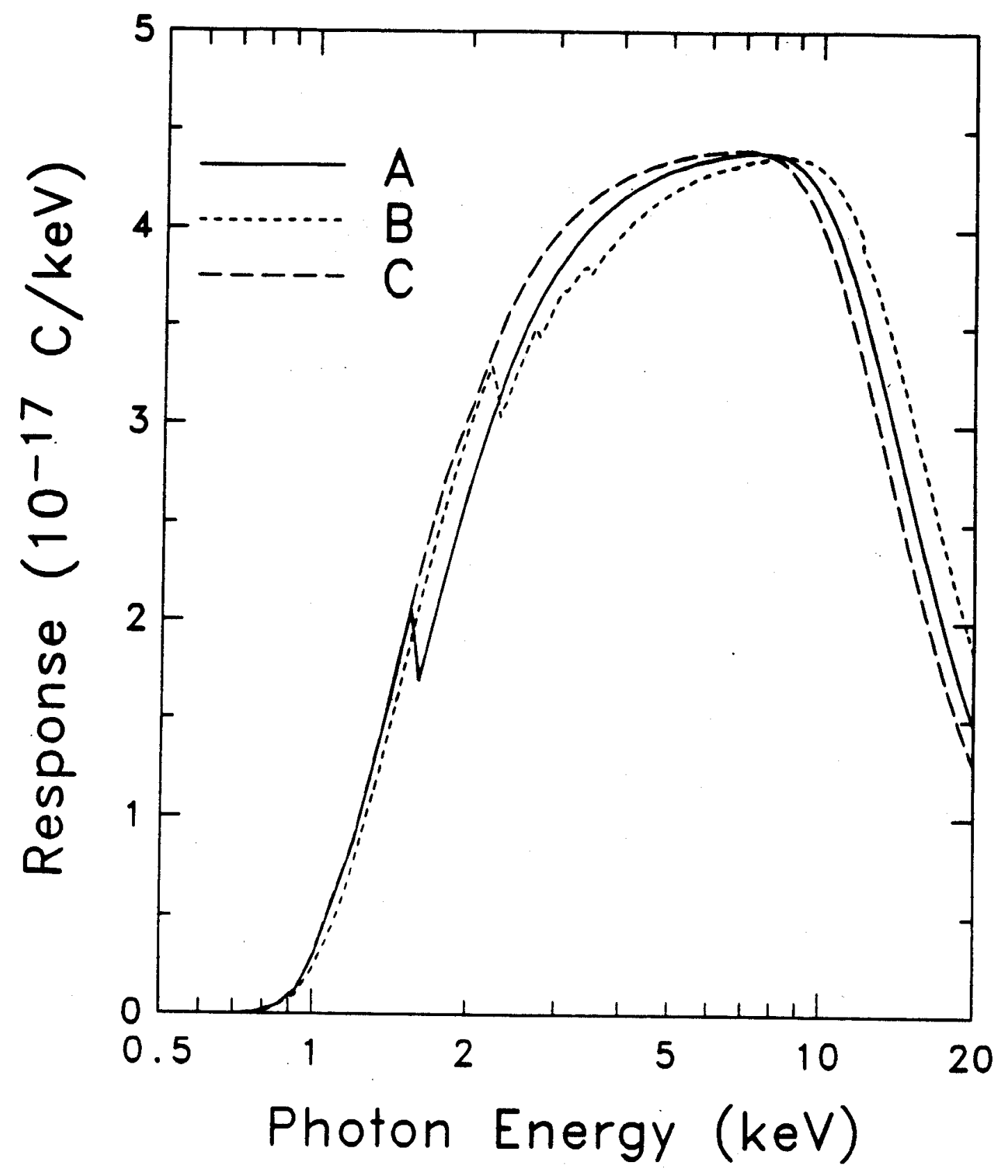

Fig. 3. The total $x$-ray response is shown for the three types of diodes, including the important effect of the $25 \mu \mathrm{m}$ beryllium window which separates the tokamak vacuum from that of the $\mathrm{x}$-ray detectors. In the low-energy region the similarity in spectral response is a result of the beryllium window. The high energy response is determined by the detector physical thickness $\left(\sim 300,400\right.$ and $250 \mu$ m respectively), not the depletion depth. ${ }^{1}$ The x-ray absorption cross sections are from Refs. 37 and 38 . 


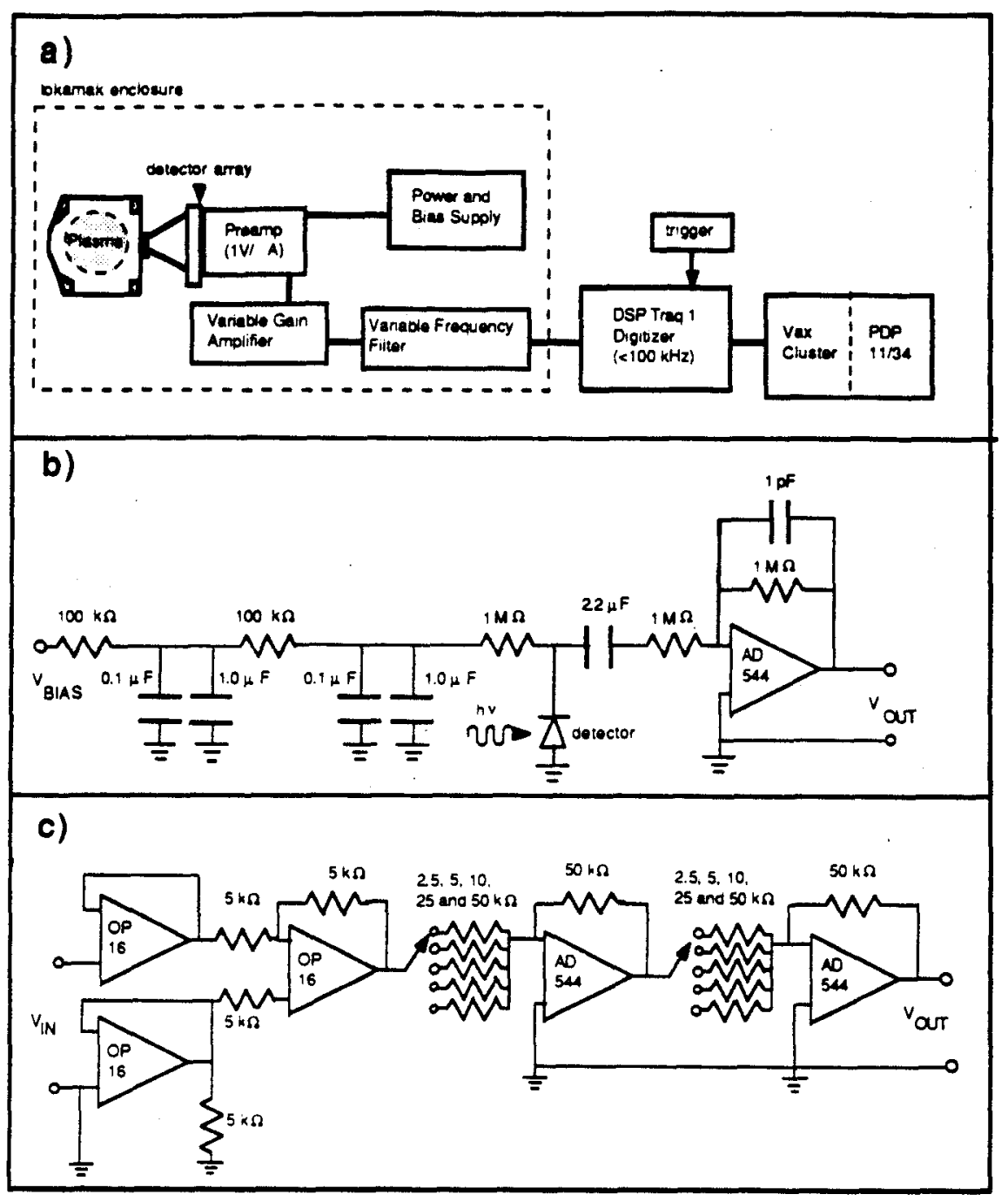

Fig. 4. a) An overall schematic of the data acquisition for Arrays B and C. (Array A has slightly different preamplifiers and amplifiers. ${ }^{31}$ ) The signal is converted from a current to a voltage in the preamplifier, subsequently it is amplified, filtered, and finally sent to the TEXT data room for digitization and storage. b) The preamplifier circuit used for Array $\mathrm{B}$ is $\mathrm{AC}$-coupled to allow the bias voltage to be easily varied and to avoid long time scale and DC changes in signal levels. (The Array C preamp is also AC-coupled, but the bias voltage is a fixed $-15 \mathrm{~V}$.) With an AC-coupled circuit no compensation for the leakage current is required. The typical pulse length on TEXT is $500 \mathrm{~ms}$, and the RC time constant of the preamp is $\simeq 2 M \Omega \times 2.2 \mu F=4.4$ s. c) The amplifier circuit can achieve voltage amplification of $1,2,4,5,10,20,25,40,50,100,200$, and 400 , by combining different gains of the two stages. The signal level may then be easily set to match the dynamic range of $=5 \mathrm{~V}$ of the digitizers. For channels with the weakest signals (the outer-viewing channels in Fig. 1) the maximum gain used is rarely above 25. 

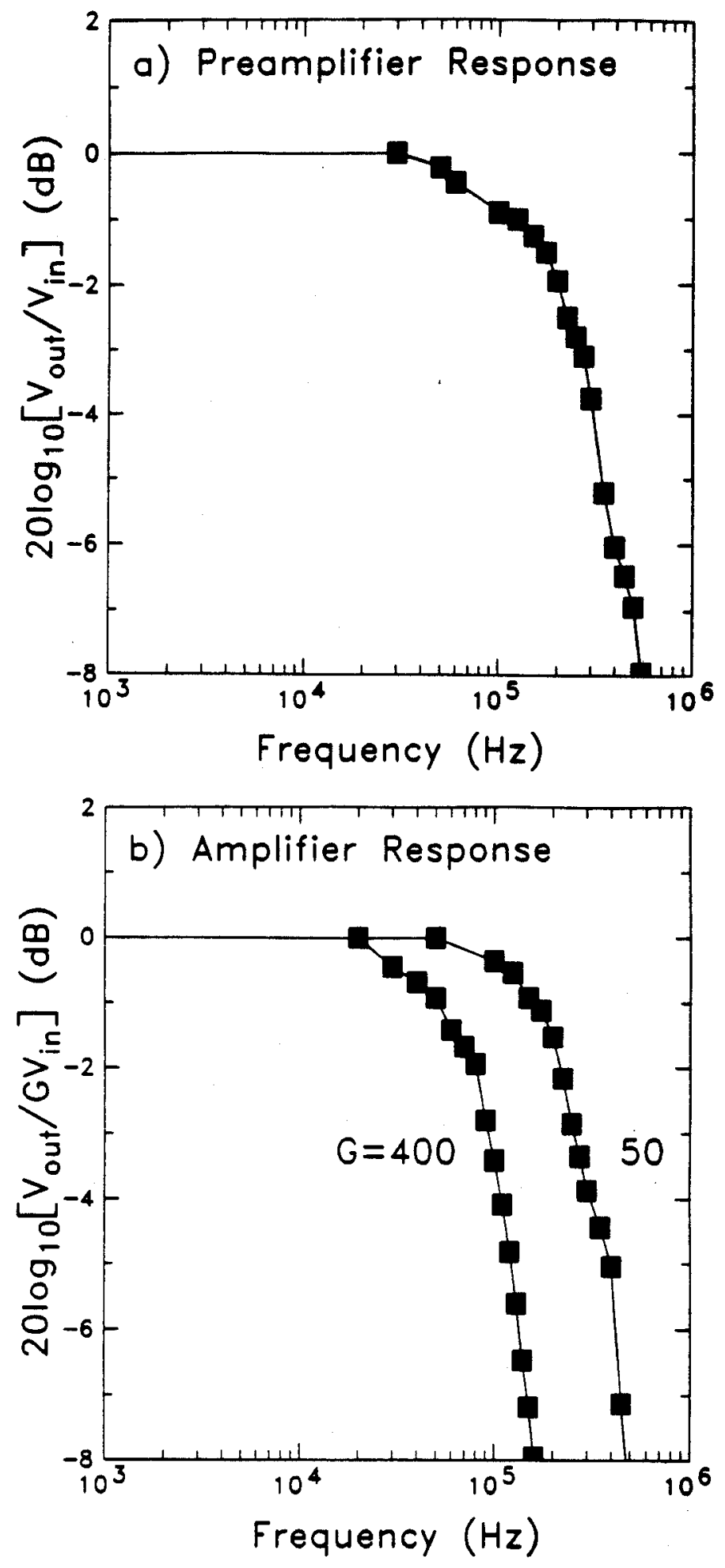

Fig. 5. The frequency response of the electronics used for data processing: a) the preamplifier response has a $3 \mathrm{~dB}$ rolloff at $\sim 250 \mathrm{kHz}$; and b) the amplifier response for gains of 50 and 400 have $3 \mathrm{~dB}$ rolloffs of $\sim 250 \mathrm{kHz}$ and $100 \mathrm{kHz}$ respectively. Since the highest gain used is rarely above 25 , the high frequency cutoff for all channels is always above 100 $\mathrm{kHz}$. 


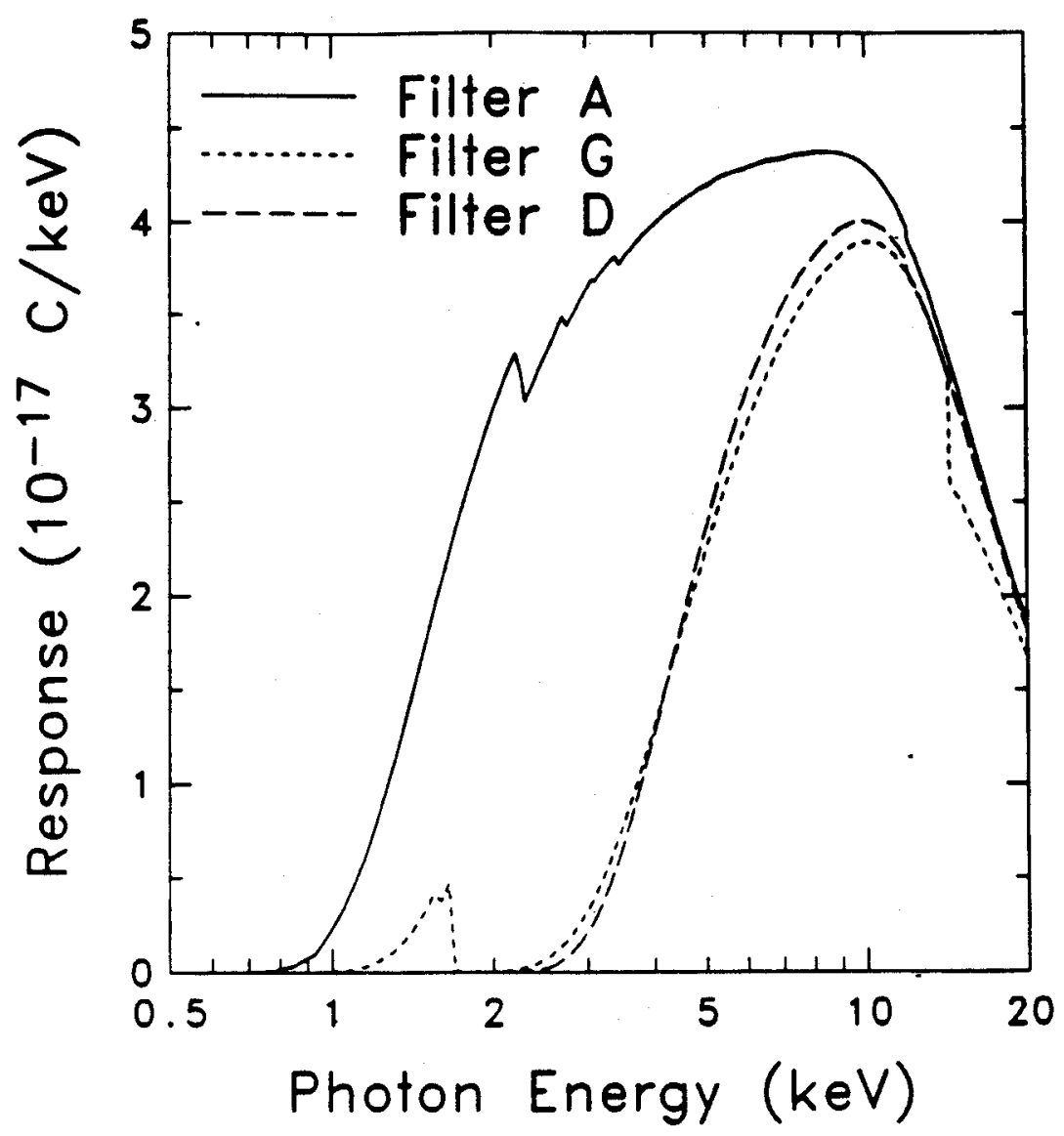

Fig. 6. The response of the flter/detector combinations used for measuring the charge state distribution of injected aluminum are shown (also used for the data of Fig. 7). The low-energy response of the detectors is determined by the three different filters, and the high energy response is determined mainly by the physical thickness of the silicon detectors. ${ }^{1}$ The three filters are comprised of $4.6 \mathrm{mg} / \mathrm{cm}^{2}$ beryllium (Filter A); $4.6 \mathrm{mg} / \mathrm{cm}^{2}$ beryllium and $1.52 \mathrm{mg} / \mathrm{cm}^{2}$ krypton (Filter $\mathrm{G}$ ); and $4.6 \mathrm{mg} / \mathrm{cm}^{2}$ beryllium plus $30.5 \mathrm{mg} / \mathrm{cm}^{2}$ carbon (Filter D). The low-energy cutoffs for Filters $\mathrm{A}$ and $\mathrm{D}$ are 1.0 and $3.0 \mathrm{keV}$ respectively; Filter $\mathrm{G}$ has a small response between 1.3 and $1.675 \mathrm{keV}$, and then again above $3.0 \mathrm{keV}$. The strong L-absorption edge of Filter $\mathrm{G}$ at $1.675 \mathrm{keV}$ serves to discriminate between the He-like and $\mathrm{H}$-like resonance lines of aluminum at 1.61 and $1.73 \mathrm{keV}$ respectively. The higher energy cutoff of Filter D eliminates all the lines from these two states, so the response for this filter is mainly to the continuum recombination radiation. ${ }^{9}$ 

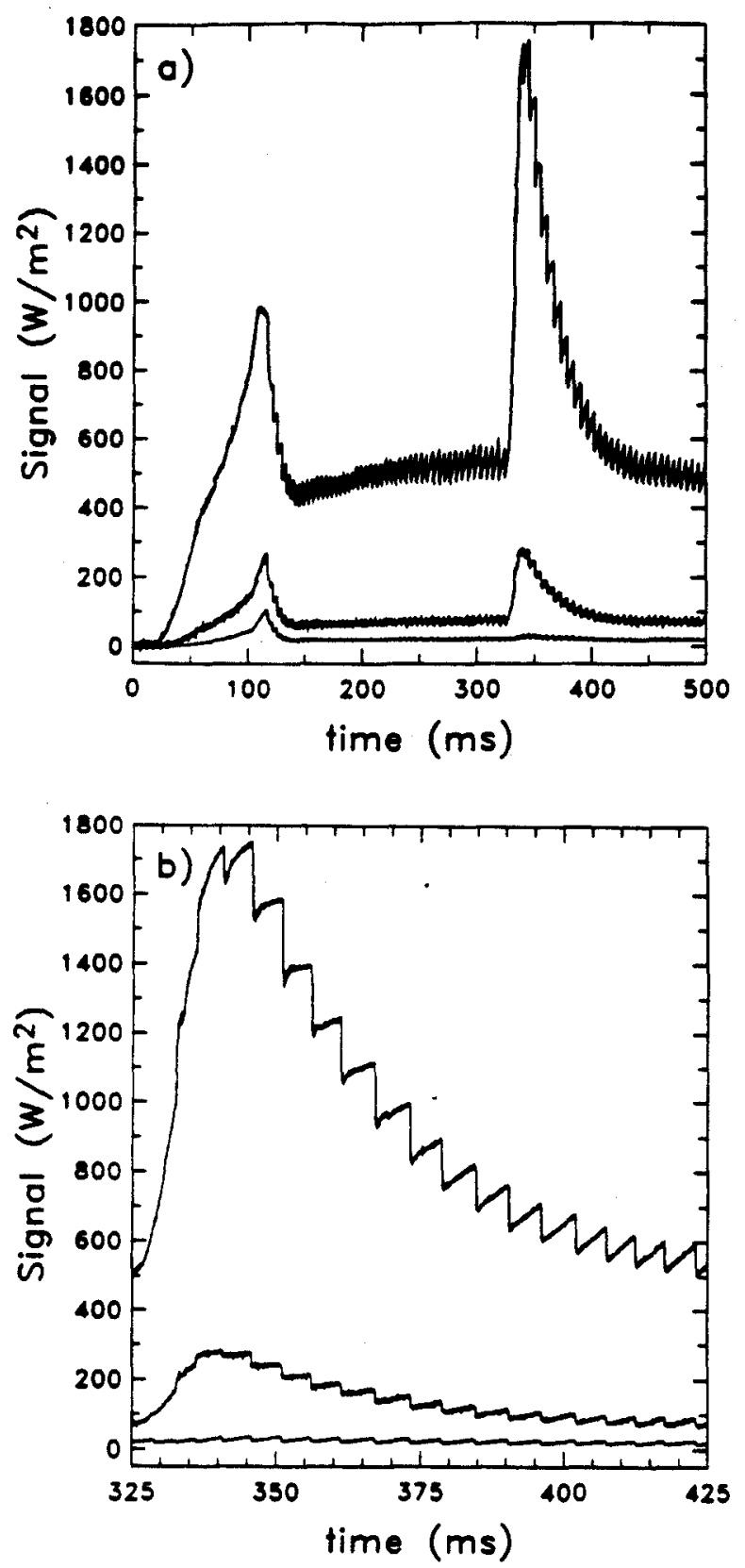

Fig. 7. Data from TEXT shot \#144004 from three differently filtered detectors viewing central chords through the plasma: an Array B channel with Filter A (top trace); an Array A channel with Filter G (middle trace); and an Array B channel with Filter D (bottom trace) (See Fig. 6 for the corresponding energy response curves.) a) Time traces throughout the entire discharge; and b) an expansion of the data around the time of aluminum injection at $325 \mathrm{~ms}$. 


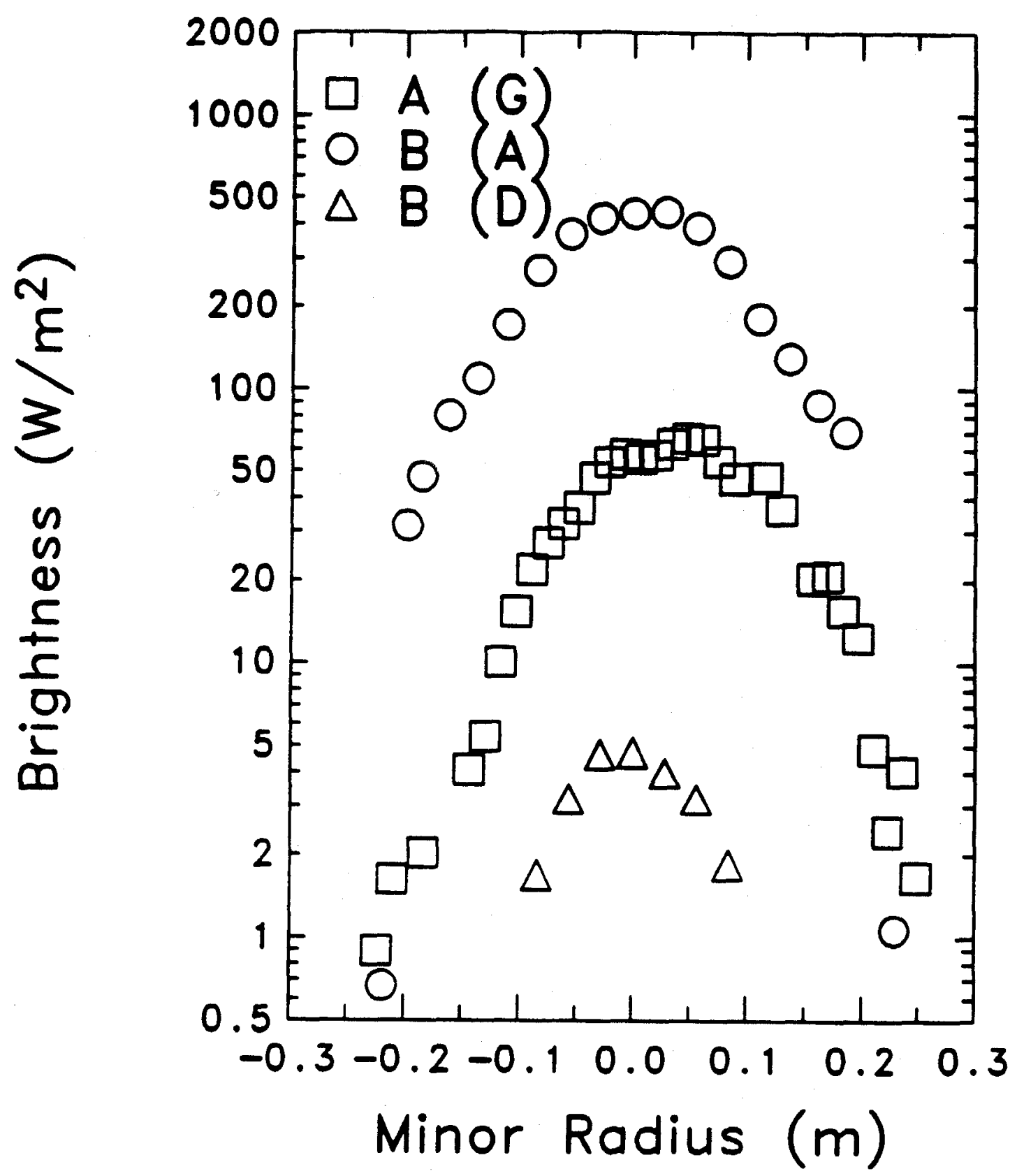

Fig. 8. a) Brightness profiles taken at $372 \mathrm{~ms}$ of shot \#144004, using the filters described in Fig. 6 (see also Fig. 7 for the time history of the central channel raw data). Note that the Array A (Filter G) profile is shifted outward due to the normal Shafronov shift, and all the profiles are approximately symmetric about their centers. Furthermore, the similarity in shape of the profiles from Filters $A$ and $G$ indicate that the He-like charge state is dominant in the plasma. 